\title{
COMPLETE LIFT OF VECTOR FIELDS AND SPRAYS TO $T^{\infty} M$
}

\author{
A. SURI AND S. RASTEGARZADEH
}

\begin{abstract}
In this paper for a given Banach, possibly infinite dimensional, manifold $M$ we focus on the geometry of its iterated tangent bundle $T^{r} M, r \in \mathbb{N} \cup\{\infty\}$. First we endow $T^{r} M$ with a canonical atlas using that of $M$. Then the concepts of vertical and complete lifts for functions and vector fields on $T^{r} M$ are defined which they will play a pivotal role in our next studies i.e. complete lift of (semi)sprays. Afterward we supply $T^{\infty} M$ with a generalized Fréchet manifold structure and we will show that any vector field or (semi)spray on $M$, can be lifted to a vector field or (semi)spray on $T^{\infty} M$. Then, despite of the natural difficulties with non-Banach modeled manifolds, we will discuss about the ordinary differential equations on $T^{\infty} M$ including integral curves, flows and geodesics. Finally, as an example, we apply our results to the infinite dimensional case of manifold of closed curves.

Keywords: Vertical and complete lift, semispray, spray, geodesic,, Fréchet manifolds, Banach manifold, Manifold of closed curves.
\end{abstract}

\section{Contents}

Introduction

1. Preliminaries

2. Complete and vertical lifts

2.1. Vertical and complete lifts of functions and vector fields

2.2. Semisprays and their lifts

3. Complete lift of vector fields and sprays to $T^{\infty} M$

3.1. Flow of $X^{c_{\infty}}$

3.2. Lift of semisprays to $T^{\infty} M$

3.3. Lift of sprays to $T^{\infty} M$

4. Applications and examples

References

\section{INTRODUCTION}

Lift of the geometric objects to tangent bundles had witnessed a wide interest due to the works of Miron [15], Bucataru and Dahl [5], Morimoto [16], Yano, Kobayashi and Ishihara [23, 24] and Suri [20, 21].

On the other hand, one of the most important generalizations of ordinary differential equations to manifolds is the class of second order differential

Date: 2015.

2000 Mathematical Subject Classification. Primary 58A05 ; 58B20 ; Secondary 70 G45 
equations (SODEs) or (semi)sprays. A semispray on $M$ is a vector filed $S$ on $T M$ such that, if $\pi: T M \longrightarrow M$ denotes the canonical projection of $T M$ on $M$, then $\pi_{*} \circ S=i d_{M}$ and the homogeneity requirement of order 2, leads us to the concept of sprays [5, 13]. In fact semisprays and sprays provide a geometric structure to study the curves on the manifold $M$ which solve special systems of SODEs with remarkable geometric meanings [5, 7, 13. Moreover, the setting of semisprays provides a unified framework for studying geodesics in Riemann, Finsler and Lagrange geometries (See [13, [6] and the references therein.)

However, most of these materials are proved for the finite dimensional case and there are a few researcher which they follow the formalism of Abraham etc. 2], Lang [13, Hamilton [11] or Kriegel and Michor [14. In this paper, for a Banach modeled manifold $M$, first we endow the iterated tangent bundle $T^{r} M, r \in \mathbb{N}$, with a canonical atlas using that of $M$. Then following the direction of [5], the concepts of vertical and complete lifts for functions and vector fields on $T^{r} M$ are defined which they will play a pivotal role in our next studies i.e. complete lifts of semisprays and sprays.

Afterward, using the algebraic tool "projective limit", which is compatible with our geometric setting, we will try to lift vector fields, semisprays and sprays to $T^{\infty} M$. More precisely we show that the iterated lifts of vector field (or semispray) form a projective system and their limits satisfy the appropriate conditions for vector fields (or semisprays) on the Fréchet manifold $T^{\infty} M$. Despite of a lack of general solvability and uniqueness theorem for ordinary differential equations for non-Banach modeled Fréchet manifolds [11, we will prove an existence theorem for the flow of lifted vector fields on $T^{\infty} M$. As a consequence we show that for the given initial values, there exists a unique geodesic with respect to the lifted (semi)sprays on $T^{\infty} M$ with non-trivial lifetime.

However, our attempt to define the lift of functions (and forms) from the base manifold $M$ to $T^{\infty} M$ fails. In fact the sequence of iterated complete (and vertical) lifts of functions, does not satisfy the appropriate conditions for projective limits of map (see remark 3.9).

Apart from the manifold structure of $T^{\infty} M$, we can define complete and vertical lifts of functions, vector fields, semisprays and sprays form $T^{\infty} M$ to $T T^{\infty} M$ similar to that in section 2 . One of the main goals of this article is to build a class of concrete example in Fréchet geometry for which we can find geodesics (and flows) with nontrivial lifetime (see also remark 3.14).

Finally, as an advantage of working with infinite dimensional Banach manifolds, we will apply our results to the Banach manifolds of closed curves.

Through this paper all the maps and manifolds are assumed to be smooth and the base manifold $M$ is partitionable.

\section{Preliminaries}

Let $M$ be a smooth manifold modeled on the Banach space $\mathbb{E}$ and $\pi_{0}$ : $T M \longrightarrow M$ be its tangent bundle. We remind that $T M=\bigcup_{x \in M} T_{x} M$ such that $T_{x} M$ consists of all equivalent classes of the form $[c, x]$ where

$$
c \in C_{x}=\{c:(\epsilon, \epsilon) \longrightarrow M ; \epsilon>0, c \text { is smooth and } c(0)=x\},
$$


under the equivalence relation

$$
c_{1} \sim_{x} c_{2} \Longleftrightarrow c_{1}^{\prime}(0)=c_{2}^{\prime}(0)
$$

for $c_{1}, c_{2} \in C_{x}$. The projection map $\pi_{0}: T M \longrightarrow M$, maps $[c, x]$ onto $x$. If $\mathcal{A}_{0}=\left\{\left(\phi_{\alpha_{0}}:=\phi_{\alpha}, U_{\alpha_{0}}:=U_{\alpha}\right) ; \alpha \in I\right\}$ is an atlas for $M$, then we have the canonical atlas $\mathcal{A}_{1}=\left\{\left(\phi_{\alpha_{1}}:=D \phi_{\alpha_{0}}, U_{\alpha_{1}}:=\pi_{0}^{-1}\left(U_{\alpha}\right)\right) ; \alpha \in I\right\}$ for $T M$ where

$$
\begin{aligned}
\phi_{\alpha_{1}}: \pi_{0}^{-1}\left(U_{\alpha_{0}}\right) \longrightarrow & \longrightarrow U_{\alpha_{0}} \times \mathbb{E} \\
{[c, x] } & \longmapsto\left(\left(\phi_{\alpha_{0}} \circ c\right)(0),\left(\phi_{\alpha_{0}} \circ c\right)^{\prime}(0)\right) .
\end{aligned}
$$

Inductively one can define an atlas for $T^{r} M:=T\left(T^{r-1} M\right), r \in \mathbb{N}$, by

$$
\mathcal{A}_{r}:=\left\{\left(\phi_{\alpha_{r}}:=D \phi_{\alpha_{r-1}}, U_{\alpha_{r}}:=\pi_{r-1}^{-1}\left(U_{\alpha_{r-1}}\right)\right) ; \alpha \in I\right\}
$$

for which $\pi_{r-1}: T^{r} M \longrightarrow T^{r-1} M$ is the natural projection. The model spaces for $T M$ and $T^{r} M$ are $\mathbb{E}_{1}:=\mathbb{E}^{2}$ and $\mathbb{E}_{r}:=\mathbb{E}^{2^{r}}$ respectively. We add here to the convention that $T^{0} M=M$.

\section{Complete and vertical lifts}

The vertical and complete lift of geometric objects has been studied by many authors (see e.g [23], 24], [5], [6] and the references therein). For a comprehensive treatment in the finite dimensional case and for references to the extensive literature on the subject one may refer to the paper [5] by Bucataru and Dahl.

In this section we introduce the concepts of vertical and complete lifts of functions and vector fields from $T^{r} M, r \in \mathbb{N} \cup\{0\}$, to $T^{r+1} M$ for the Banach manifold $M$.

Set $\kappa_{1}:=i d_{T M}$ and for $r \geq 2$ consider the canonical involution $\kappa_{r}$ : $T^{r} M \longrightarrow T^{r} M$ which satisfies $\partial_{t} \partial_{s} \gamma(t, s)=\kappa_{r} \partial_{s} \partial_{t} \gamma(t, s)$, for any smooth map $\gamma:(-\epsilon, \epsilon)^{2} \longrightarrow T^{r-2} M$. If we consider $T^{r-2} M$ as a smooth manifold modeled on $\mathbb{E}_{r-2}$, then the charts of $T^{r-1} M, T^{r} M$ and $T^{r+1} M$ take their values in $\mathbb{E}_{r-1}=\mathbb{E}_{r-2}^{2}, \mathbb{E}_{r}=\mathbb{E}_{r-2}^{4}$ and $\mathbb{E}_{r+1}=\mathbb{E}_{r-2}^{8}$ respectively. It is easy to check that the local representation of $\kappa_{r}$ is given by

$$
\begin{aligned}
\kappa_{r \alpha}:=\phi_{\alpha_{r}} \circ \kappa_{r} \circ \phi_{\alpha_{r}}^{-1}: U_{\alpha_{r-2}} \times \mathbb{E}_{r-2}^{3} & \longrightarrow U_{\alpha_{r-2}} \times \mathbb{E}_{r-2}^{3} \\
(x, y, X, Y) & \longmapsto(x, X, y, Y) .
\end{aligned}
$$

Setting $D^{2}=D D$, the relations

$$
\begin{aligned}
\kappa_{r}^{2} & =I d_{T^{r} M} \\
\pi_{r} \circ D \kappa_{r} & =\kappa_{r} \circ \pi_{r} \\
D \pi_{r-1} & =\pi_{r} \circ \kappa_{r+1} \\
D^{2} \pi_{r-1} \circ \kappa_{r+2} & =\kappa_{r+1} \circ D^{2} \pi_{r-1} \\
D \pi_{r-1} \circ \pi_{r+1} & =\pi_{r} \circ D^{2} \pi_{r-1},
\end{aligned}
$$

$r \in \mathbb{N}$, as they are listed in [6, 5], are valid in our framework too. 
2.1. Vertical and complete lifts of functions and vector fields. There are two known lifts for functions and vector fields due to [23] and [5]. Most of the materials of this section are modified versions of those form [5].

Definition 2.1. Suppose that $r \geq 0$ and $f \in C^{\infty}\left(T^{r} M\right)$. Then the vertical lift of $f$ is the smooth function $f^{v} \in C^{\infty}\left(T^{r+1} M\right)$ defined by

$$
f^{v}(\xi)=\left(f \circ \pi_{r} \circ \kappa_{r+1}\right)(\xi), \quad \xi \in T^{r+1} M .
$$

If $r=0$, then equation (6) implies that $f^{v}=f \circ \pi_{0}$ and for $r \geq 1$, the equations (2) and (4) imply that $f^{v}=f \circ D \pi_{r-1}$. Moreover, the local representation of $f^{v}$ is

$$
\begin{aligned}
f_{\alpha}^{v}:=f^{v} \circ \phi_{\alpha_{r+1}}^{-1}: U_{\alpha_{r-1}} \times \mathbb{E}_{r-1}^{3} & \longrightarrow \mathbb{R} \\
(x, y, X, Y) & \longmapsto f_{\alpha}(x, X)
\end{aligned}
$$

where $f_{\alpha}:=f \circ \phi_{\alpha_{r}}^{-1}: U_{\alpha_{r-1}} \times \mathbb{E}_{r-1} \longrightarrow \mathbb{R}$.

Definition 2.2. For $r \geq 0$ and $f \in C^{\infty}\left(T^{r} M\right)$ the complete lift of $f$ is the smooth function $f^{c} \in C^{\infty}\left(T^{r+1} M\right)$ defined by

$$
f^{c}(\xi)=d f \circ \kappa_{r+1}(\xi), \quad \xi \in T^{r+1} M .
$$

Clearly for $r=0, f^{c}(\xi)=d f(\xi)$. If $r \geq 1$, then the local representation of $f^{c}$ is

$$
\begin{aligned}
f_{\alpha}^{c}:=f^{c} \circ \phi_{\alpha_{r+1}}^{-1}: U_{\alpha_{r-1}} \times \mathbb{E}_{r-1}^{3} & \longrightarrow \mathbb{R} \\
(x, y, X, Y) & \longmapsto \partial_{1} f(x, X) y+\partial_{2} f(x, X) Y
\end{aligned}
$$

where $\partial_{i}, i=1,2$, stands for the partial derivative with respect to the i'th variable.

Following the formalism of [5] we define the concepts of vertical and complete lifts for vector field in the Banach case.

Definition 2.3. Let $r \geq 0$ and $\mathbf{A}: T^{r} M \longrightarrow T^{r+1} M$ be a vector field. The vertical lift of $\mathbf{A}$ is the vector field $\mathbf{A}^{v}: T^{r+1} M \longrightarrow T^{r+2} M$ defined by the following relation

(9) $\mathbf{A}^{v}(\xi)=\left.D \kappa_{r+1} \circ \partial_{s}\left(\kappa_{r+1}(\xi)+s \mathbf{A} \circ \pi_{r} \circ \kappa_{r+1}(\xi)\right)\right|_{s=0}, \quad \xi \in T^{r+1} M$.

$\mathbf{A}^{v}$ is a vector field since

$$
\begin{aligned}
\pi_{r+1} \circ \mathbf{A}^{v}(\xi) & =\left.\pi_{r+1} \circ D \kappa_{r+1} \circ \partial_{s}\left(\kappa_{r+1}(\xi)+s \mathbf{A} \circ \pi_{r} \circ \kappa_{r+1}(\xi)\right)\right|_{s=0} \\
& =\left.\kappa_{r+1} \circ \pi_{r+1} \circ \partial_{s}\left(\kappa_{r+1}(\xi)+s \mathbf{A} \circ \pi_{r} \circ \kappa_{r+1}(\xi)\right)\right|_{s=0} \\
& =\kappa_{r+1} \circ \kappa_{r+1}(\xi) \\
& =\xi
\end{aligned}
$$

Locally on the chart $\left(U_{\alpha_{r}}, \phi_{\alpha_{r}}\right)$, suppose that the local representation of $\mathbf{A}$ be given by

$$
\begin{aligned}
\mathbf{A}_{\alpha}:=\phi_{\alpha_{r+1}} \circ \mathbf{A} \circ \phi_{\alpha_{r}}^{-1}: U_{\alpha_{r-1}} \times \mathbb{E}_{r-1} & \longrightarrow U_{\alpha_{r-1}} \times \mathbb{E}_{r-1}^{3} \\
(x, y) & \longmapsto\left(x, y ; A_{\alpha}(x, y), B_{\alpha}(x, y)\right)
\end{aligned}
$$

where $A_{\alpha}$ and $B_{\alpha}$ are smooth $\mathbb{E}_{r-1}$ valued functions on $U_{\alpha_{r}} \subseteq T^{r} M$. Then the vertical lift of $\mathbf{A}$ is the vector field $\mathbf{A}^{v} \in \mathfrak{X}\left(T^{r+1} M\right)$ with the local 
representation $\mathbf{A}_{\alpha}^{v}:=\phi_{\alpha_{r+2}} \circ \mathbf{A}^{v} \circ \phi_{\alpha_{r+1}}^{-1}$ where

$$
\begin{aligned}
\mathbf{A}_{\alpha}^{v}: U_{\alpha_{r-1}} \times \mathbb{E}_{r-1}^{3} & \longrightarrow U_{\alpha_{r-1}} \times \mathbb{E}_{r-1}^{7} \\
(x, y, X, Y) & \longmapsto\left(x, y, X, Y ; 0, A_{\alpha}(x, X), 0, B_{\alpha}(x, X)\right) .
\end{aligned}
$$

Note that $A_{\alpha}(x, X)=A_{\alpha}^{v}(x, y, X, Y)$ and $B_{\alpha}(x, X)=B_{\alpha}^{v}(x, y, X, Y)$.

Finally we introduce the concept of complete lift of a vector field which will play a key role in our next studies.

Definition 2.4. Let A : $T^{r} M \longrightarrow T^{r+1} M, r \geq 0$, be a vector field. The complete lift of $\mathbf{A}$ is the vector field $\mathbf{A}^{c}: T^{r+1} M \longrightarrow T^{r+2} M$ defined by

$$
\mathbf{A}^{c}=D \kappa_{r+1} \circ \kappa_{r+2} \circ D \mathbf{A} \circ \kappa_{r+1}
$$

Note that

$$
\begin{aligned}
\pi_{r+1} \circ \mathbf{A}^{c}(\xi) & =\pi_{r+1} \circ D \kappa_{r+1} \circ \kappa_{r+2} \circ D \mathbf{A} \circ \kappa_{r+1}(\xi) \\
& =\kappa_{r+1} \circ \pi_{r+1} \circ \kappa_{r+2} \circ D \mathbf{A} \circ \kappa_{r+1}(\xi) \\
& =\kappa_{r+1} \circ D \pi_{r} \circ D \mathbf{A} \circ \kappa_{r+1}(\xi) \\
& =\kappa_{r+1} \circ D\left(\pi_{r} \circ \mathbf{A}\right) \circ \kappa_{r+1}(\xi) \\
& =\kappa_{r+1} \circ \kappa_{r+1}(\xi) \\
& =\xi
\end{aligned}
$$

which means that $\mathbf{A}^{c}$ is a vector field (see also [5]). Moreover with the above notations the local representation of $\mathbf{A}^{c}$ is

$$
\begin{aligned}
\mathbf{A}_{\alpha}^{c}: U_{\alpha_{r-1}} \times \mathbb{E}_{r-1}^{3} & \longrightarrow U_{\alpha_{r-1}} \times \mathbb{E}_{r-1}^{7} \\
\xi=(x, y, X, Y) & \longmapsto\left(x, y, X, Y ; A_{\alpha}^{v}(\xi), A_{\alpha}^{c}(\xi), B_{\alpha}^{v}(\xi), B_{\alpha}^{c}(\xi)\right)
\end{aligned}
$$

More precisely

$$
\begin{aligned}
\mathbf{A}_{\alpha}^{c}(\xi)= & \left(x, y, X, Y ; A_{\alpha}(x, X), \partial_{1} A_{\alpha}(x, X) y+\partial_{2} A_{\alpha}(x, X) Y\right. \\
& \left., B_{\alpha}(x, X), \partial_{1} B_{\alpha}(x, X) y+\partial_{2} B_{\alpha}(x, X) Y\right)
\end{aligned}
$$

2.2. Semisprays and their lifts. For $r \geq 1$ a semispray on $T^{r-1} M$ is a vector field $S: T^{r} M \longrightarrow T^{r+1} M$ with the additional property $\kappa_{r+1} \circ S=S$ (or equivalently $D \pi_{r-1} \circ S=i d_{T^{r} M}$ ) ([5]). Considering the atlas $\mathcal{A}_{r-1}$, we observe that locally on the chart $\left(\phi_{\alpha_{r-1}}, U_{\alpha_{r-1}}\right)$ the local representation of the vector field $S$ is $S_{\alpha}:=\phi_{\alpha_{r+1}} \circ S \circ \phi_{\alpha r}^{-1}$ and

$$
\begin{aligned}
S_{\alpha}: U_{\alpha_{r-1}} \times \mathbb{E}_{r-1} & \longrightarrow U_{\alpha_{r-1}} \times \mathbb{E}_{r-1}^{3} \\
(x, y) & \longmapsto\left(x, y ; A_{\alpha}(x, y), B_{\alpha}(x, y)\right)
\end{aligned}
$$

where $A_{\alpha}, B_{\alpha}: U_{\alpha_{r-1}} \times \mathbb{E}_{r-1} \longrightarrow \mathbb{E}_{r-1}$ are smooth functions locally representing $S$. The condition $\kappa_{r+1} \circ S=S$ (or $D \pi_{r} \circ S=i d_{T^{r} M}$ ) yields that $A_{\alpha}(x, y)=y$. By convention, we set $B_{\alpha}(x, y):=-2 G_{\alpha}(x, y)$. Hence the local representation of $S$ is

$$
S_{\alpha}: U_{\alpha_{r-1}} \times \mathbb{E}_{r-1} \longrightarrow U_{\alpha_{r-1}} \times \mathbb{E}_{r-1}^{3} ;(x, y) \longmapsto\left(x, y ; y,-2 G_{\alpha}(x, y)\right) \text {. }
$$

Considering the semispray $S$ as a vector field and using the notion of complete lift for vector field, we define the complete lift $S^{c}=D \kappa_{r+1} \circ \kappa_{r+2} \circ$ $D S \circ \kappa_{r+1}$. Bucataru and Dahl in [5] proved that $S^{c}$ is a semispray. Here we state a modified version of their proof for infinite dimensional manifolds. 
Proposition 2.5. $S^{c}$ is a semispray on $T^{r} M$.

Proof. It suffices to show that $\kappa_{r+2} \circ S^{c}=S^{c}$. For $\xi=(x, y, X, Y) \in T^{r+1} M$ we have

$$
\begin{aligned}
\kappa_{r+2} \circ S^{c}(\xi)= & \kappa_{r+2} \circ D \kappa_{r+1} \circ \kappa_{r+2} \circ D S \circ \kappa_{r+1}(\xi) \\
= & \kappa_{r+2} \circ D \kappa_{r+1} \circ \kappa_{r+2} \circ D S(x, X, y, Y) \\
= & \kappa_{r+2} \circ D \kappa_{r+1} \circ \kappa_{r+2}(S(x, X), d S(x, X)(y, Y)) \\
= & \kappa_{r+2} \circ D \kappa_{r+1} \circ \kappa_{r+2}\left(x, X, X,-2 G_{\alpha}(x, X) ; y, Y, Y\right. \\
& \left.,-2 d G_{\alpha}(x, X)(y, Y)\right) \\
= & \kappa_{r+2} \circ D \kappa_{r+1}\left(x, X, y, Y ; X,-2 G_{\alpha}(x, X), Y,-2 d G_{\alpha}(x, X)(y, Y)\right) \\
= & \kappa_{r+2}\left(\kappa_{r+1}(x, X, y, Y), D \kappa_{r+1}(x, X, y, Y)\left(X,-2 G_{\alpha}(x, X), Y\right.\right. \\
& \left.,-2 d G_{\alpha}(x, X)(y, Y)\right) \\
= & \kappa_{r+2}\left(x, y, X, Y ; X, Y,-2 G_{\alpha}(x, X),-2 d G_{\alpha}(x, X)(y, Y)\right) \\
= & \left(x, y, X, Y ; X, Y,-2 G_{\alpha}(x, X),-2 d G_{\alpha}(x, X)(y, Y)\right) \\
= & \left(x, y, X, Y ; X, Y,-2 G_{\alpha}^{v}(\xi),-2 G_{\alpha}^{c}(\xi)\right) \\
= & S^{c}(\xi)
\end{aligned}
$$

which completes the proof.

Definition 2.6. A geodesic for the semispray $S$ is a smooth curve $\gamma$ : $(-\epsilon, \epsilon) \longrightarrow T^{r-1} M$ such that its canonical lift $\gamma^{\prime}$ is an integral curve for $S$ i.e. $\gamma^{\prime \prime}(t)=S\left(\gamma^{\prime}(t)\right), t \in(-\epsilon, \epsilon)$.

Locally on a chart this last means that

$$
\begin{aligned}
\left(\gamma_{\alpha}(t), \gamma_{\alpha}^{\prime}(t), \gamma_{\alpha}^{\prime}(t), \gamma_{\alpha}^{\prime \prime}(t)\right) & =S_{\alpha}\left(\left(\gamma_{\alpha}(t), \gamma_{\alpha}^{\prime}(t)\right)\right. \\
& =\left(\gamma_{\alpha}(t), \gamma_{\alpha}^{\prime}(t), \gamma_{\alpha}^{\prime}(t),-2 G_{\alpha}\left(\gamma_{\alpha}(t), \gamma_{\alpha}^{\prime}(t)\right)\right)
\end{aligned}
$$

where $\gamma_{\alpha}=\phi_{\alpha_{r-1}} \circ \gamma$. Therefore the curve $\gamma$ is a geodesic for the semispray $S$ if and only if it satisfies the following second order ordinary differential equation (SODE)

$$
\gamma_{\alpha}^{\prime \prime}(t)+2 G_{\alpha}\left(\gamma_{\alpha}(t), \gamma_{\alpha}^{\prime}(t)\right)=0 ; \alpha \in I
$$

which are known as geodesic equations with respect to $S$.

Remark 2.7. For a Riemannian manifold with its canonical metric spray ([13]), equations (11) coincide with the usual geodesic equations.

\section{Complete lift of Vector fields And sprays to $T^{\infty} M$}

The geometry of $T^{r} M, r \in \mathbb{N}$, and lifting of geometric objects to this bundle was studied by many authors (See [5, 23] and the references therein). However, no extension in the direction of $T^{\infty} M$ has appeared in the literatures. The aim of this section is to lift a vector field $X \in \mathfrak{X}(T M)$, to a vector field $X^{c_{\infty}} \in \mathfrak{X}\left(T T^{\infty} M\right)$.

To assess the benefits of this lift, we will consider the interesting cases of semisprays and sprays. In fact we will try to lift a (semi)sprays form $M$ to $T^{\infty} M$. 
In order to introduce $T^{\infty} M$ we will consider it as an appropriate limit (projective or inverse limit) of the finite factors $T^{i} M$. To make our exposition as self-contained as possible, we state some preliminaries about projective limits of sets, topological vector spaces, manifolds and vector bundles from [3, 10, 18].

Let $\mathbb{I}$ be a directed set. Remind that $\mathbb{I}$ is directed if it is an ordered set with the reflexive, transitive and anti-symmetric order " $\leq$ " such that for any $i, j \in \mathbb{I}$ there exists $k \in \mathbb{I}$ with $i \leq k$ and $j \leq k$. Let $\left\{S_{i}\right\}_{i \in \mathbb{I}}$ be a family of nonempty sets. Moreover suppose that for $j \geq i$ in $\mathbb{I}$, there exists a map $f_{j i} ; S_{j} \longrightarrow S_{i}$, known as the connecting morphism, such that $f_{i i}=i d_{S_{i}}$ and $f_{j i} \circ f_{k j}=f_{k i}$ for $k \geq j \geq i$ in $\mathbb{I}$. Then $\mathcal{S}=\left\{S_{i}, f_{j i}\right\}_{i, j \in \mathbb{I}}$ is called a projective system of sets. The projective limit of this system, defined by a universal property, always exists and is (set theoretically) isomorphic to a subset of $\prod_{i \in \mathbb{I}} S_{i}$ [18]. More precisely the projective limit of the system $\mathcal{S}$, denoted by $\lim _{\longleftarrow} S_{i}$, contains those elements $\left(x_{i}\right)_{i \in I} \in \prod_{i \in \mathbb{I}} S_{i}$ for which $f_{j i}\left(x_{j}\right)=x_{i}$ for all $i, j \in \mathbb{I}$ with $j \geq i$.

Let $\left\{\mathbb{E}_{i}\right\}_{i \in \mathbb{I}}$ be family of topological vector spaces and $\rho_{j i}: \mathbb{E}_{j} \longrightarrow \mathbb{E}_{i}$, $j \geq i$ be continuous and linear. Then the projective family $\left\{\mathbb{E}_{i}, \rho_{j i}\right\}_{i \in \mathbb{I}}$ is called a projective systems of topological vector spaces. Note that every Fréchet space is isomorphic to a projective limit of a countable family of Banach spaces (18, p. 53).

In the sequel suppose that $\mathbb{I}=\mathbb{N}$ and consider the family $\mathcal{M}=\left\{M_{i}\right.$, $\left.\varphi_{j i}\right\}_{i, j \in \mathbb{N}}$ where $M_{i}, i \in \mathbb{N}$, is a manifold modeled on the Banach space $\mathbb{E}_{i}$ and $\varphi_{j i}: M_{j} \longrightarrow M_{i}$ is a differentiable map for $j \geq i$. Moreover suppose that the following conditions hold.

i) The model spaces $\left\{\mathbb{E}_{i}, \rho_{j i}\right\}_{i, j \in \mathbb{N}}$ form a projective system of topological vector spaces.

ii) For any $x=\left(x_{i}\right)_{i \in \mathbb{N}} \in M=\lim M_{i}$ there exists a projective family of charts $\left\{\left(U_{i}, \phi_{i}\right)\right\}$ such that $x_{i} \in \overleftarrow{U_{i}} \subseteq M_{i}$ and for $j \geq i, \rho_{j i} \circ \phi_{j}=\phi_{i} \circ \varphi_{j i}$ [10.

In this case $M:=\lim M_{i}$ may be considered as a generalized Fréchet manifold modeled on the Fréchet space $\mathbb{E}=\lim _{\leftarrow} \mathbb{E}_{i}$ with the atlas $\left\{\left(\lim _{i}, \lim \phi_{i}\right)\right\}$.

Finally, suppose that $\left(E_{i}, p_{i}, M_{i}\right), i \in \mathbb{N}$, be a family of a Banach vector bundles with the fibres of type $\mathbb{E}^{i}$ respectively. Moreover suppose that $\left\{\left(E_{i}, f_{j i}\right)\right\}_{i \in \mathbb{N}}$ and $\left\{\mathbb{E}^{i}, \lambda_{j i}\right\}_{i, j \in \mathbb{N}}$ are projective systems of manifolds and Banach spaces respectively. Then, the system $\left\{\left(E_{i}, p_{i}, M_{i}\right),\left(f_{j i}, \varphi_{j i}, \lambda_{j i}\right)\right\}_{i \in \mathbb{N}}$ is called a strong projective system of Banach vector bundles on $\left\{\left(M_{i}, \varphi_{j i}\right)\right\}_{i \in \mathbb{N}}$ if;

for any $\left(x_{i}\right)_{i \in \mathbb{N}}$, there exists a projective system of trivializations $\left(U_{i}, \tau_{i}\right)$ (here $\tau_{i}: p_{i}{ }^{-1}\left(U_{i}\right) \longrightarrow U_{i} \times \mathbb{E}_{i}$ are local diffeomorphisms which are linear on fibres) of $\left(E_{i}, p_{i}, M_{i}\right)$ such that $x_{i} \in M_{i}, U=\lim U^{i}$ is open in $M$ and $\left(\varphi_{j i} \times \lambda_{j i}\right) \circ \tau_{j}=\tau_{i} \circ f_{j i}$ for all $i, j \in \mathbb{N}$ with $j \geq i$.

Remark 3.1. Let $\left\{M_{i}, \varphi_{j i}\right\}$ be a projective family of Banach manifolds. It is known that the family $\left\{T M_{i}, q_{j i}\right\}_{i, j \in \mathbb{N}}$ also form a projective system of Banach manifolds (vector bundles) [10, 3] where the connecting morphisms 
are given by

$$
\begin{aligned}
q_{j i}: T M_{j} & \longrightarrow T M_{i} \\
{[f, x]^{j} } & \longmapsto\left[\varphi_{j i} \circ f, \varphi_{j i} \circ x\right]^{i}
\end{aligned}
$$

and $[., .]^{i}$ stands for the tangent vectors in $T M_{i}$.

For any element $\left(x_{i}\right)_{i \in \mathbb{N}}$ in $M:=\lim M_{i}$ consider the family of charts $\left\{\left(U_{\alpha}^{i}, \phi_{\alpha}^{i}\right)\right\}_{i \in \mathbb{N}}$ of $\left\{M_{i}\right\}_{i \in \mathbb{N}}$, around $\left(x_{i}\right)_{i \in \mathbb{N}}$ such that $\left(U_{\alpha}=\varliminf_{\alpha} U_{\alpha}^{i}, \phi_{\alpha}=\right.$ $\left.\lim _{\alpha} \phi_{\alpha}^{i}\right)$ is a chart of $M$. Fix the atlas $\left\{\left(U_{\alpha}=\lim _{\longleftarrow} U_{\alpha}^{i}, \phi_{\alpha}=\lim _{\longleftarrow}^{i}\right)\right\}_{\alpha \in \mathbb{I}}$ for $\overleftarrow{M}_{\text {. }}$

Using this atlas we can define a generalized vector bundle structure on $\pi: T M \longrightarrow M[10,3]$. More precisely $\left\{U_{\alpha}^{i}, \tau_{\alpha}^{i}\right\}_{i \in \mathbb{N}}$ form a projective system of trivializations for the strong projective family of vector bundles $\left\{\left(T M_{i}, \pi^{i}, M_{i}\right),\left(q_{j i}, \varphi_{j i}, \rho_{j i}\right)\right\}_{j \geq i}$ where

$$
\begin{aligned}
\tau_{\alpha}^{i}:\left(\pi^{i}\right)^{-1}\left(U_{\alpha}^{i}\right) & \longrightarrow U_{\alpha}^{i} \times \mathbb{E}^{i} \\
{\left[f_{i}, x_{i}\right]^{i} } & \longmapsto\left(x_{i},\left(\varphi_{\alpha}^{i} \circ f_{i}\right)^{\prime}(0)\right) .
\end{aligned}
$$

Regarding the fact $\varphi_{j i} \circ \pi^{j}=\pi^{i} \circ q_{j i}$, for $j \geq i$, we define the limit map $\pi_{M}:=\lim \pi_{i}: T M \longrightarrow M$. The trivializations of $T M$ are given by $\tau_{\alpha}=$ $\lim _{\alpha}^{i}: \pi^{-1}\left(U_{\alpha}\right) \longrightarrow U_{\alpha} \times \mathbb{F}$ where $\mathbb{F}=\lim _{\leftarrow} \mathbb{E}_{i}[10,3]$.

In order to reduce our computations, we state the following lemma.

Lemma 3.2. Let $\left\{M_{i}, \varphi_{j i}\right\}_{i, j \in \mathbb{N}}$ and $\left\{N_{i}, \psi_{j i}\right\}_{i, j \in \mathbb{N}}$ be two projective system of manifolds with the limits $M=\lim M_{i}$ and $N=\lim N_{i}$. The system $\left\{f_{i}: M_{i} \longrightarrow N_{i}\right\}$ is a projective system of maps if and only if $\psi_{i+1, i} \circ f_{i+1}=$ $f_{i} \circ \phi_{i+1, i}$, where $\psi_{i+1} i:=\psi_{i+1, i}$ and $\phi_{i+1} i:=\phi_{i+1, i}$, for any $i \in \mathbb{N}$.

Proof. Let $f_{i} \circ \phi_{i+1, i}=\psi_{i+1, i} \circ f_{i+1}$ for any $i \in \mathbb{N}$. Then for the natural number $j \in \mathbb{N}$ we have

$$
\begin{aligned}
\psi_{j i} \circ f_{j} & =\left(\psi_{i+1, i} \circ \ldots \circ \psi_{j-1, j-2} \circ \psi_{j, j-1}\right) \circ f_{j} \\
& =\left(\psi_{i+1, i} \circ \ldots \circ \psi_{j-1, j-2}\right) \circ\left(\psi_{j, j-1} \circ f_{j}\right) \\
& =\left(\psi_{i+1, i} \circ \ldots \circ \psi_{j-1, j-2}\right) \circ\left(f_{j-1} \circ \phi_{j, j-1}\right) \\
& =\psi_{i+1, i} \circ \ldots \circ f_{j-2} \circ \phi_{j-1, j-2} \circ \phi_{j, j-1} \\
& \vdots \\
& =f_{i} \circ\left(\phi_{i+1, i} \circ \ldots \circ \phi_{j-1, j-2} \circ \phi_{j, j-1}\right) \\
& =f_{i} \circ \phi_{j i} .
\end{aligned}
$$

The converse is trivial.

Remark 3.3. The family $\left\{T^{i} M\right\}_{i \in \mathbb{N}}$ with the connecting morphisms $\pi_{i+1, i}:=$ $\pi_{i}$ form a projective system of Banach manifolds. The connecting morphisms for the model spaces are $\rho_{i+1, i}: \mathbb{E}_{i+1}=\mathbb{E}^{2^{i+1}} \longrightarrow \mathbb{E}_{i}=\mathbb{E}^{2^{i}}$; $(x, y) \in \mathbb{E}_{i}^{2} \longmapsto x \in \mathbb{E}_{i}, i \in \mathbb{N}$, and the projective family of charts is given by $\left\{\left(U_{\alpha_{i}}, \phi_{\alpha_{i}}\right)\right\}_{i \in \mathbb{N}}$ with the limit $\left(U_{\alpha_{\infty}}=\lim _{\longleftarrow} U_{\alpha_{i}}, \phi_{\alpha_{\infty}}=\lim \phi_{\alpha_{i}}\right)$. The limit of this family is denoted by $T^{\infty} M:=\lim T^{i} M$ and it is called the infinite order iterated tangent bundle of $\overleftarrow{M}$. 
Proposition 3.4. Let $X \in \mathfrak{X}(T M)$ and set

$$
X^{c_{1}}:=X^{c}=D \kappa_{2} \circ \kappa_{3} \circ D X \circ \kappa_{2}
$$

and, for $j \in \mathbb{N}, X^{c_{j}}:=\left(X^{c_{j-1}}\right)^{c}$ with $X^{c_{0}}=X$. The following statements hold true.

i) $D^{2} \pi_{0} \circ X^{c_{1}}=X \circ D \pi_{0}$.

ii) For any $i \in \mathbb{N}$

$$
D^{2} \pi_{i} \circ X^{c_{i+1}}=X^{c_{i}} \circ D \pi_{i} .
$$

iii) For any $i, j \in \mathbb{N}$ with $j \geq i$ we have

$$
D^{2} \pi_{j, i} \circ X^{c_{j}}=X^{c_{i}} \circ D \pi_{j, i},
$$

where $\pi_{j, i}:=\pi_{i} \circ \pi_{i+1} \circ \ldots \circ \pi_{j-1}: T^{j} M \longrightarrow T^{i} M$.

Proof. i.) Using equations (2) with $r=1$ and (12) we see that

$$
\begin{aligned}
D D \pi_{0} \circ X^{c_{1}} & =D\left(\pi_{1} \circ \kappa_{2}\right) \circ X^{c_{1}} \\
& =D \pi_{1} \circ D \kappa_{2} \circ D \kappa_{2} \circ \kappa_{3} \circ D X \circ \kappa_{2} \\
& =D \pi_{1} \circ \kappa_{3} \circ D X \circ \kappa_{2} \\
& =\pi_{2} \circ D X \circ \kappa_{2}=X \circ \pi_{1} \circ \kappa_{2}=X \circ D \pi_{0} .
\end{aligned}
$$

ii.) For the natural number $i$ we see that

$$
\begin{aligned}
D^{2} \pi_{i} \circ X^{c_{i+1}} & =D\left(\pi_{i+1} \circ \kappa_{i+2}\right) \circ X^{c_{i+1}} \\
& =D \pi_{i+1} \circ D \kappa_{i+2} \circ D \kappa_{i+2} \circ \kappa_{i+3} \circ D X^{c_{i}} \circ \kappa_{i+2} \\
& =D \pi_{i+1} \circ \kappa_{i+3} \circ D X^{c_{i}} \circ \kappa_{i+2} \\
& =\pi_{i+2} \circ D X^{c_{i}} \circ \kappa_{i+2} \\
& =X^{c_{i}} \circ \pi_{i+1} \circ \kappa_{i+2} \\
& =X^{c_{i}} \circ D \pi_{i}
\end{aligned}
$$

which proves the second assertion. Equivalently, the following diagram is commutative.

$$
\begin{array}{ccccccc}
T\left(T^{i+1} M\right) & \stackrel{D \pi_{i}}{\longrightarrow} & T\left(T^{i} M\right) & \cdots & T(T M) & \stackrel{D \pi_{0}}{\longrightarrow} & T M \\
X^{c_{i+1}} \downarrow & & \downarrow X^{c_{i}} & & \downarrow X^{c_{1}} & & \downarrow X \\
T^{2}\left(T^{i+1} M\right) & \stackrel{D^{2} \pi_{i}}{\longrightarrow} & T^{2}\left(T^{i} M\right) & \cdots & T^{2}(T M) & \stackrel{D^{2} \pi_{0}}{\longrightarrow} & T^{2} M
\end{array}
$$

iii.) The last part of the proposition can be deduced from part (ii) and Lemma 3.2. More precisely we have the following commutative diagram.

$$
\begin{array}{ccc}
T\left(T^{j} M\right) & \stackrel{D \pi_{j, i}}{\longrightarrow} & T\left(T^{i} M\right) \\
X^{c_{j}} \downarrow & & \downarrow X^{c_{i}} \\
T^{2}\left(T^{j} M\right) & \stackrel{D^{2} \pi_{j, i}}{\longrightarrow} & T^{2}\left(T^{i} M\right)
\end{array}
$$

As a consequence, $\left\{X^{c_{i}}\right\}_{i \in \mathbb{N}}$ form a projective system maps from $\left\{T\left(T^{i} M\right)\right.$,

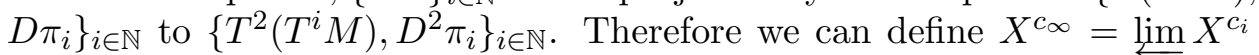
from $T T^{\infty} M=\lim _{\longleftarrow}\left\{T\left(T^{i} M\right), D \pi_{i}\right\}$ to $T^{2}\left(T^{\infty} M\right)=\lim _{\longleftarrow}\left\{T^{2}\left(T^{i} M\right), D^{2} \pi_{i}\right\}$ (See also [10] p. 5 or [3] p. 6 ). 
Theorem 3.5. $X^{c_{\infty}}=\lim _{\longleftarrow} X^{c_{i}}$ exists and is a vector field on $T T^{\infty} M$.

Proof. The family of vector bundles

$$
\left\{\left(E_{i}, p_{i}, N_{i}\right):=\left(T^{2}\left(T^{i} M\right), \pi_{i+1}, T\left(T^{i} M\right)\right)\right\}_{i \in \mathbb{N}}
$$

form a strong projective systems of vector bundles (in the sense of [3] Def. $4.1)$. More precisely, the fibre type of the bundle $\left(E_{i}, p_{i}, N_{i}\right), i \in \mathbb{N}$, is $\mathbb{E}^{2^{i+1}}$ with the corresponding connecting morphism

$$
\begin{aligned}
\lambda_{i+1, i}:\left(\mathbb{E}^{2^{i-1}}\right)^{4}=\mathbb{E}^{2^{i+1}} & \longrightarrow=\left(\mathbb{E}^{2^{i-1}}\right)^{2}=\mathbb{E}^{2^{i}} \\
(x, y, X, Y) & \longmapsto(x, X) .
\end{aligned}
$$

As a consequence we get, $\phi_{\alpha_{i+1}} \circ D^{2} \pi_{i-1}=\left(D \pi_{i-1} \times \lambda_{i+1, i}\right) \circ \phi_{\alpha_{i+2}}$ where $\phi_{\alpha_{i+k}}, k=1,2$, are the local charts of $T^{i+k} M$. Then, according to proposition 4.4 of [3], $T^{2} T^{\infty} M:=\lim _{\longleftarrow} T^{2}\left(T^{i} M\right)$ admits a generalized Fréchet vector bundle structure over $T T^{\infty} M:=\lim T\left(T^{i} M\right)$.

However, $\pi_{i+1} \circ D^{2} \pi_{i}=D \pi_{i} \circ \pi_{i+2}$ for any $i \in \mathbb{N}([\underline{6}$, p. 2123).

$$
\begin{array}{ccccccc}
T\left(T^{i+1} M\right) & \stackrel{D \pi_{i}}{\longrightarrow} & T\left(T^{i} M\right) & \cdots & T(T M) & \stackrel{D \pi_{0}}{\longrightarrow} & T M \\
\pi_{i+2} \uparrow & & \uparrow \pi_{i+1} & & \uparrow \pi_{2} & & \uparrow \pi_{1} \\
T^{2}\left(T^{i+1} M\right) & \stackrel{D^{2} \pi_{i}}{\longrightarrow} & T^{2}\left(T^{i} M\right) & \cdots & T^{2}(T M) & \stackrel{D^{2} \pi_{0}}{\longrightarrow} & T^{2} M
\end{array}
$$

This last means that the limit map $\varliminf_{i m} \pi_{i+1}$ exists and locally maps $(x, y, X$, $Y) \in\left(T^{2} \phi_{\alpha_{\infty}}\right)^{-1}\left(U_{\alpha_{\infty}}\right)$ onto $(x, y)$. As a consequence $\lim _{i+1}$ exists and $\lim _{i+1}: T^{2}\left(T^{\infty} M\right) \longrightarrow T\left(T^{\infty} M\right)$ is equal to $\pi_{T T^{\infty} M}$. Moreover the resulting vector bundle is a generalized vector bundle isomorphic to $\pi_{T T^{\infty} M}$ : $T^{2}\left(T^{\infty} M\right) \longrightarrow T\left(T^{\infty} M\right)$ (the tangent bundle of $T\left(T^{\infty} M\right)$ ).

According to [5], if $X^{c_{i-1}} \in \mathfrak{X}\left(T\left(T^{i-1} M\right)\right)$ then $X^{c_{i}} \in \mathfrak{X}\left(T\left(T^{i} M\right)\right)$. Consequently, for any $\xi \in T\left(T^{\infty} M\right)$, we have

$$
\pi_{T T^{\infty} M} \circ X^{c_{\infty}}(\xi)=\lim _{\longleftarrow} \pi_{i+1} \circ X^{c_{\infty}}(\xi)=\left(\pi_{i+1} \circ X^{c_{i}}\left(\xi_{i}\right)\right)_{i \in \mathbb{N}}=\left(\xi_{i}\right)_{i \in \mathbb{N}}
$$

which means that $X^{c_{\infty}}$ is a vector field on $T T^{\infty} M$.

3.1. Flow of $X^{c_{\infty}}$. In closing this section, despite of the lack of a general solvability and uniqueness theorem for ordinary differential equations on non-Banach Fréchet manifolds ([11]), we prove an existence theorem for the flow of $X^{c_{\infty}}$.

Let $i \in \mathbb{N}, X \in \mathfrak{X}\left(T^{i} M\right)$ and $F: \mathfrak{D}(X) \subseteq T^{i} M \times \mathbb{R} \longrightarrow T^{i} M$ be its flow (see e.g. [13]). We remark that for $\xi \in T^{i} M, F(\xi,):. I(\xi) \subseteq \mathbb{R} \longrightarrow T^{i} M$; $t \longmapsto F_{t}(\xi):=F(\xi, t)$, is an integral curve of $X$ with $F_{\xi}(0)=\xi$ and $I(\xi)$ is its maximal domain (lifetime) in $\mathbb{R}$.

Suppose that $F: \mathfrak{D}(X) \longrightarrow T^{i} M$ and $F^{c}: \mathfrak{D}\left(X^{c}\right) \longrightarrow T^{i+1} M$ are the flows of $X$ and $X^{c}$ respectively. According to [5] theorem 3.6, we have $\left(D \pi_{i-1} \times i d_{\mathbb{R}}\right) \mathfrak{D}\left(X^{c}\right)=\mathfrak{D}(X)$ and for any $(\xi, t) \in \mathfrak{D}\left(X^{c}\right), F_{t}^{c}(\xi)=\kappa_{i+1} \circ$ $D F_{t} \circ \kappa_{i+1}(\xi)$ where $D F_{t}$ is the differential of the map $F_{t}: \xi \longmapsto F_{t}(\xi)$. Note that the model spaces of manifolds in [5] are finite dimensional Euclidean spaces. However, the proof of theorem 3.6. is valid for the Banach modeled manifolds as well.

Let $X \in \mathfrak{X}(T M)$ and $F: \mathfrak{D}(X) \subseteq T M \times \mathbb{R} \longrightarrow T M$ be its flow. For $i \in \mathbb{N}$, set $X^{c_{i}}=\left(X^{c_{i-1}}\right)^{c}$ and $F^{c_{i}}=\left(F^{c_{i-1}}\right)^{c}$. 
Theorem 3.6. For $i \in \mathbb{N}$, suppose that $F^{c_{i}}: \mathfrak{D}\left(X^{c_{i}}\right) \subseteq T\left(T^{i} M\right) \times \mathbb{R} \longrightarrow$ $T\left(T^{i} M\right)$ be the flow of $X^{c_{i}}$. Then the following statements hold true.

i.) $\left\{\mathfrak{D}\left(X^{c_{i}}\right) \subseteq T\left(T^{i} M\right) \times \mathbb{R},\left.\left(D \pi_{i} \times i d_{\mathbb{R}}\right)\right|_{\mathfrak{D}\left(X^{c_{i}}\right)}\right\}_{i \in \mathbb{N}}$ form a projective system of open sets.

ii.) $F^{c_{\infty}}=\lim _{\longleftarrow} F^{c_{i}}$ exists.

iii.) $F^{c_{\infty}}$ is the flow of $X^{c_{\infty}}$.

Proof. i.) Since $\left\{T^{i} M, \pi_{j i}\right\}$ is a projective system, then $\left\{T\left(T^{i} M\right), D \pi_{j i}\right\}$ is a projective system with the limit isomorphic to $T\left(T^{\infty} M\right)$. It is easy to check that $\left.\left\{\mathfrak{D}\left(X^{c_{i}}\right) \subseteq T^{(} T^{i} M\right) \times \mathbb{R},\left.\left(D \pi_{j i} \times i d_{\mathbb{R}}\right)\right|_{\mathfrak{D}\left(X^{c_{i}}\right)}\right\}$ also form a projective system. Set $\mathfrak{D}\left(X^{c_{\infty}}\right):=\lim \mathfrak{D}\left(X^{c_{i}}\right) \cdot \mathfrak{D}\left(X^{c_{\infty}}\right)$ is an open subset of $T\left(T^{\infty} M\right) \times \mathbb{R}$ with respect to the projective topology of $T\left(T^{\infty} M\right)$.

ii.) For any $i \in \mathbb{N}$ and any $\xi=(x, y, X, Y) \in T\left(T^{i+1} M\right)$ we have

$$
\begin{aligned}
D \pi_{i} \circ F^{c_{i+1}}(t, \xi) & =D \pi_{i} \circ F_{t}^{c_{i+1}}(x, y, X, Y) \\
& =D \pi_{i} \circ \kappa_{i+2} \circ D F_{t}^{c_{i}} \circ \kappa_{i+2}(x, y, X, Y) \\
& =\left(\pi_{i+1} \circ \kappa_{i+2}\right) \circ \kappa_{i+2} \circ D F_{t}^{c_{i}}(x, X, y, Y) \\
& =\pi_{i+1}\left(F_{t}^{c_{i}}(x, X), d F_{t}^{c_{i}}(x, X)(y, Y)\right) \\
& =F_{t}^{c_{i}}(x, X)
\end{aligned}
$$

and

$$
F^{c_{i}} \circ\left(D \pi_{i} \times i d_{\mathbb{R}}\right)(\xi, t)=F_{t}^{c_{i}} \circ D \pi_{i}(x, y, X, Y)=F_{t}^{c_{i}}(x, X)
$$

that is the following diagram is commutative.

$$
\begin{array}{ccc}
\mathfrak{D}\left(X^{c_{i+1}}\right) & \stackrel{F^{c_{i+1}}}{\longrightarrow} & T\left(T^{i+1} M\right) \\
D \pi_{i} \times i d_{\mathbb{R}} \downarrow & & \downarrow D \pi_{i} \\
\mathfrak{D}\left(X^{c_{i}}\right) & \stackrel{F^{c_{i}}}{\longrightarrow} & T\left(T^{i} M\right)
\end{array}
$$

As a consequence of lemma 3.2, $F^{c_{\infty}}:=\lim _{\longleftarrow} F^{c_{i}}: \lim \mathfrak{D}\left(X^{c_{i}}\right) \longrightarrow T\left(T^{\infty} M\right)$ can be defined.

iii.) Finally, we claim that $F^{c_{\infty}}: \mathfrak{D}\left(X^{c_{\infty}}\right):=\lim \mathfrak{D}\left(X^{c_{i}}\right) \longrightarrow T\left(T^{\infty} M\right)$ is the flow of $X^{c_{\infty}}$. For the latter we note that $F_{\xi}^{\overleftarrow{c_{\infty}}}: I(\xi) \subseteq \mathbb{R} \longrightarrow T T^{\infty} M$ is an integral curve for $X^{c_{\infty}}$, for all $(\xi, t) \in \mathfrak{D}\left(X^{c_{\infty}}\right)$, since

$$
\frac{d}{d t} F_{\xi}^{c_{\infty}}(t)=\lim _{\longleftarrow} \frac{d}{d t} F_{\xi_{i}}^{c_{i}}(t)=\varliminf_{\longleftarrow} X^{c_{i}}\left(F_{\xi_{i}}^{c_{i}}(t)\right)=X^{c_{\infty}}\left(F_{\xi}^{c_{\infty}}(t)\right) .
$$

It may be worth reminding the reader that a vector field is called complete if, all of its integral curves admit the lifetime $(-\infty,+\infty)$.

Corollary 3.7. If $X$ is a complete vector field on $M$, then $X^{c_{\infty}}$ is a complete vector field on $T\left(T^{\infty} M\right)$ too.

With the notations as in theorem 3.6, we have the following useful result.

Corollary 3.8. For any $\xi=\left(\xi_{i}\right)_{i \in \mathbb{N}} \in T\left(T^{\infty} M\right)$, the unique integral curve $F_{\xi}^{c_{\infty}}=\lim _{\longleftarrow} F_{\xi_{i}}^{c_{i}}: I(\xi) \longrightarrow T\left(T^{\infty} M\right)$ with $F_{\xi}^{c_{\infty}}(0)=\xi^{\infty}$ exists.

Remark 3.9. Let $f \in C^{\infty}(M)$. Set $f^{c_{1}}=f^{c}$ and $f^{c_{i}}=f^{c_{i-1}}$. Then according to definition 2.2 ,

$$
f^{c_{i}}=d\left(f^{c_{i-1}}\right) \circ \kappa_{i}
$$


If we consider the projective systems $\left\{T^{i} M, \pi_{i}\right\}_{i \in \mathbb{N}}$ and $\left\{\mathbb{R}, i d_{\mathbb{R}}\right\}_{i \in \mathbb{N}}$ with the limits $T^{\infty} M=\lim T^{i} M$ and $\mathbb{R}=\lim \mathbb{R}$ respectively, then $f^{c_{i}} \circ \pi_{i} \neq$ $i d_{\mathbb{R}} \circ f^{c_{i+1}}$. In fact for any $(x, y, X, Y) \in \overleftarrow{T}^{i+1} M$

$$
f^{c_{i}} \circ \pi_{i}(x, y, X, Y)=f^{c_{i}}(x, y)
$$

while

$$
\begin{aligned}
i d_{\mathbb{R}} \circ f^{c_{i+1}}(x, y, X, Y) & =d f^{c_{i}} \circ \kappa_{i+1}(x, y, X, Y)=d f^{c_{i}}(x, X, y, Y) \\
& =\partial_{1} f^{c_{i}}(x, X) y+\partial_{2} f^{c_{i}}(x, X) Y .
\end{aligned}
$$

As a consequence, the sequence $\left\{f^{c_{i}}\right\}_{i \in \mathbb{N}}$ is not a projective system of maps and consequently we can not define $f^{c_{\infty}}$ as the $\operatorname{limit} \lim f^{c_{i}}$. Similarly one can show that, the iterated vertical lift of functions and lift of forms, generally, do not form projective systems. We refer to 1 p. 39, 4 p. 204 and [22] p. 549 for special classes of functions and forms on projective limits of manifolds.

3.2. Lift of semisprays to $T^{\infty} M$. In this section, for a given semispray on $M$ we shall try to lift it to a semispray on $T^{\infty} M$.

Proposition 3.10. For a given semispray $S$ on $M$ its iterated complete lift $S^{c_{\infty}}$ is a semispray on $T^{\infty} M$.

Proof. Since $S$ is a vector field on $T M$, then proposition 3.4 ensures us that $\left\{S^{c_{i}}: T\left(T^{i} M\right) \longrightarrow T^{2}\left(T^{i} M\right)\right\}$ forms a projective system of vector fields with the limit $S^{c_{\infty}} \in \mathfrak{X}\left(T\left(T^{\infty} M\right)\right)$.

Proposition 2.5 shows that for any $i \in \mathbb{N}, S^{c_{i}}=\left(S^{c_{i-1}}\right)^{c}$ is a semispray on $T^{i} M$.

Moreover one can check that $\left\{D \pi_{i}\right\}_{i \in \mathbb{N}}$ form a projective system maps with the limit $D \pi_{T^{\infty} M}$ where $\pi_{T^{\infty} M}=\lim \pi_{i}: T T^{\infty} M \longrightarrow T^{\infty} M$ is the tangent bundle of $T^{\infty} M$. Consequently for any $\xi \in T^{\infty} M$,

$$
D \pi_{\infty} \circ S^{c_{\infty}}(\xi)=\lim _{\longleftarrow}\left(D \pi_{i} \circ S^{c_{i}}\left(\xi_{i}\right)\right)=\lim _{\longleftarrow}\left(\xi_{i}\right)=\xi,
$$

which completes the proof.

Let $S$ be a semispray on $T^{\infty} M$. Keeping the formalism of section 2.2 , a geodesic with respect to $S$ is a curve $\gamma:(-\epsilon, \epsilon) \subset \mathbb{R} \longrightarrow T^{\infty} M$ such that $\gamma^{\prime \prime}(t)=S\left(\gamma^{\prime}(t)\right), \epsilon>0$ and $t \in(-\epsilon, \epsilon)$. Now suppose that $S$ be a semispray on $M$ and $S^{c_{\infty}}$ be its lift to $T^{\infty} M$ given by proposition 3.10 .

Theorem 3.11. For any $x=\left(x_{i}\right)_{i \in \mathbb{N}} \in T^{\infty} M, \xi=\left(\xi_{i}\right)_{i \in \mathbb{N}} \in T_{x} T^{\infty} M$, there exists a unique geodesic $\gamma_{\xi}:(-\epsilon, \epsilon) \longrightarrow T^{\infty} M$ for $S^{c_{\infty}}$ such that $\gamma_{\xi}(0)=x$, $\gamma_{\xi}^{\prime}(0)=\xi$. Moreover $\gamma_{\xi}=\lim \gamma_{\xi_{i}}$ where $\gamma_{\xi_{i}}:(-\epsilon, \epsilon) \longrightarrow T\left(T^{i} M\right)$ is a geodesic of $S^{c_{i}}$ with $\gamma_{\xi_{i}}(0)=x_{i}$ and $\gamma_{\xi_{i}}^{\prime}(0)=\xi_{i}, i \in \mathbb{N}$.

Proof. According to corollary 3.8 there exists a unique curve $F_{\xi}^{c_{\infty}}:(-\epsilon, \epsilon) \longrightarrow$ $T\left(T^{\infty} M\right)$ such that $\frac{d}{d t} F_{\xi}^{c_{\infty}}(t)=S^{c_{\infty}}\left(F_{\xi}^{c_{\infty}}(t)\right), F_{\xi}^{c_{\infty}}(0)=\xi$ and $F_{\xi}=\lim F_{\xi_{i}}^{c_{i}}$.

Set $\gamma_{\xi}=\pi_{T^{\infty} M} \circ F_{\xi}:(-\epsilon, \epsilon) \longrightarrow T^{\infty} M$. We claim that $\gamma_{\xi}$ is the desired geodesic. Since

$$
\begin{aligned}
\frac{d}{d t} \gamma_{\xi}(t) & =\frac{d}{d t}\left(\pi_{T^{\infty} M} \circ F_{\xi}^{c_{\infty}}\right)(t)=D \pi_{T^{\infty} M} \circ \frac{d}{d t} F_{\xi}^{c_{\infty}}(t) \\
& =D \pi_{T^{\infty} M} \circ S^{c_{\infty}}\left(F_{\xi}^{c_{\infty}}(t)\right) \\
& =F_{\xi}^{c_{\infty}}(t)
\end{aligned}
$$


and $F_{\xi}^{c_{\infty}}$ is the integral curve of $S^{c_{\infty}}$, we conclude that

$$
\frac{d^{2}}{d t^{2}} \gamma_{\xi}(t)=S^{c_{\infty}}\left(\frac{d}{d t} \gamma_{\xi}(t)\right)
$$

Moreover $\gamma_{\xi}(0)=\pi_{T^{\infty} M} \circ F_{\xi}^{c_{\infty}}(0)=\pi_{T^{\infty} M}(\xi)=x$ and

$$
\begin{aligned}
\left.\frac{d}{d t} \gamma_{\xi}(t)\right|_{t=0} & =\left.\frac{d}{d t}\left(\pi_{T^{\infty} M} \circ F_{\xi}^{c_{\infty}}\right)(t)\right|_{t=0}=D \pi_{T^{\infty} M} \circ \frac{d}{d t} F_{\xi}^{c_{\infty}}(t)_{t=o} \\
& =D \pi_{T^{\infty} M} \circ S^{c_{\infty}}\left(F_{\xi}^{c_{\infty}}(t)\right)_{t=0} \\
& =D \pi_{T^{\infty} M} \circ S^{c_{\infty}}(\xi) \\
& =\xi .
\end{aligned}
$$

3.3. Lift of sprays to $T^{\infty} M$. There is a very close relations between linear connections and a special type of semisprays known as sprays [5, 13, 7, 19].

Definition 3.12. A semispray $S$ on $T^{i} M$ is called a (2-homogeneous) spray if for any $\alpha \in I, \lambda \in \mathbb{R}$ and $(x, \xi) \in U_{\alpha_{i}} \times \mathbb{E}_{i}, G_{\alpha}(x, \lambda \xi)=\lambda^{2} G_{\alpha}(x, \xi)$.

Proposition 3.13. The following statements hold true.

i) The complete lift of a spray is a spray.

ii) If $S$ is a spray on $M$, then $S^{c_{\infty}}$ is a spray on $T^{\infty} M$.

Proof. i.) Let $S$ be a spray on $T^{i} M$. Then, $S$ is a semispray. According to proposition 2.5, $S^{c}$ is a semispray on $T^{i+1} M$. Now, it suffices to show that the local components of $S^{c}$ are 2-homogeneous. Locally the semispray $S^{c}$ maps $\xi=(x, y, X, Y) \in T\left(T^{i+1} M\right)$ to

$$
\left(x, y, X, Y ; X, Y,-2 G_{\alpha}^{v}(\xi),-2 G_{\alpha}^{c}(\xi)\right) .
$$

However, for any $\lambda \in \mathbb{R}$ and $\xi \in T\left(T^{i+1} M\right)$,

$$
G_{\alpha}^{v}(x, y, \lambda X, \lambda Y)=G_{\alpha}(x, \lambda X)=\lambda^{2} G_{\alpha}(x, X)=\lambda^{2} G_{\alpha}^{v}(x, y, X, Y),
$$

and

$$
\begin{aligned}
G_{\alpha}^{c}(x, y, \lambda X, \lambda Y) & =d G_{\alpha}(x, \lambda X)(y, \lambda Y)=\left.\frac{d}{d t} G_{\alpha}(x+t y, \lambda(X+t Y))\right|_{t=0} \\
& =\left.\frac{d}{d t} \lambda^{2} G_{\alpha}(x+t y, X+t Y)\right|_{t=0} \\
& =\left.\lambda^{2} \frac{d}{d t} G_{\alpha}(x+t y, X+t Y)\right|_{t=0} \\
& =\lambda^{2} d G_{\alpha}(x, X)(y, Y) \\
& =\lambda^{2} G_{\alpha}^{c}(x, y, X, Y) .
\end{aligned}
$$

ii.) Due to proposition $3.10 S^{c_{\infty}}$ is a semispray on $T^{\infty} M$ with the local components $G_{\alpha}^{\infty}:=\lim _{\longleftarrow} G_{\alpha}^{i}$ which maps $\left(\left(x_{i}\right)_{i \in \mathbb{N}},\left(y_{i}\right)_{i \in \mathbb{N}}\right) \in U_{\alpha}^{\infty} \times \mathbb{E}_{\infty}$ to

$$
\left(x, y, y, G_{\alpha}^{\infty}(x, y)\right)
$$

Part one implies that for any $i \in \mathbb{N}, S^{c_{i}}$ is a spray on $T^{i} M$. Let $\left\{G_{\alpha}^{i}\right\}$ be the family of local components of $S^{c_{i}}$. Then,

$$
G_{\alpha}^{\infty}(x, \lambda y)=\lim _{\longleftarrow} G_{\alpha}^{i}\left(x_{i}, \lambda y_{i}\right)=\lambda^{2} \lim _{\longleftarrow} G_{\alpha}^{i}\left(x_{i}, y_{i}\right)=\lambda^{2} G_{\alpha}^{\infty}(x, y)
$$


for any $\lambda \in \mathbb{R}$ and $(x, y) \in U_{\alpha}^{\infty} \times \mathbb{E}_{\infty}$.

Remark 3.14. Suppose that at any step we have a metric spray $S^{c_{i}}$ with a compatible Riemannian metric $g_{i}$ (for more details see [13]). Then, the meaning of a geodesic for the spray $S^{c_{\infty}}$ on $T^{\infty} M$ would be (a local) length minimizer of the sup and sum metrics, in the topological sense (see [17] p. 1485, def. 3.5 and section 5). More precisely at any stage we have a metric space $\left(T^{i} M, d_{i}\right)$, where $d_{i}$ is the metric induced by $g_{i}$. Now, if $\gamma=\lim \gamma_{i}$ is a geodesic of $S^{c_{\infty}}$, then at every step we have a minimizer $\gamma_{i}$ of $d_{i}$. As a consequence $\gamma$ is a minimizer of the sup and sum metrics that is, the length minimizers on $T^{\infty} M$ are geodesics of $S^{c_{\infty}}$

\section{Applications and examples}

In this section we state an examples to reveal the benefits of working with Banach manifolds and our results.

Example 4.1. Let $(M, g)$ be an $n$-dimensional Riemannian manifold and $I=[0,1]$. An $H^{1}$ loop $c: I \longrightarrow \mathbb{R}^{n}$ is an absolutely continuous curve for which $\dot{c}(t)$ exists almost everywhere and $\dot{c}$ is square integrable and $c(0)=$ $c(1)$ (12] p. 159). A loop $c: I \longrightarrow M$ is called of class $H^{1}$ if, for any chart $(U, \phi)$ of $M$ the mapping $\phi \circ c: I^{\prime} \subseteq c^{-1}(U) \longrightarrow \mathbb{R}^{n}$ is $H^{1}$. Then $H^{1}(I, M)$, formed by the loops $c: I \longrightarrow M$ of class $H^{1}$, admits a Hilbert manifold structure modeled on the Hilbert space $\mathbb{H}:=H^{1}\left(c^{*} T M\right)$ [9, 12].

The space of vector fields along $c$ is isomorphic with the space of sections $\Gamma\left(c^{*} T M\right)$ of the pullback $c^{*} T M$ and it is identified with the tangent space $T_{c} H^{1}(I, M)$. The scalar product on $T_{c} H^{1}(I, M)$ is given by $\langle u, v\rangle_{c}=$ $\int_{0}^{1} g(u(t), v(t)) d t+\int_{0}^{1} g\left(\frac{D}{d t} u(t), \frac{D}{d t} v(t)\right) d t$ for any $u, v \in T_{c} H^{1}(I, M)$. (For a detailed study about the geometry of $H^{1}(I, M)$ we refer to [12.) However, one may consider the $C^{k}$ maps and construct a Banach manifold structure space using the sup-norm instead of using $L^{2}$ norms [8].

Let $f: M \longrightarrow N$ be a differentiable map between finite dimensional Riemannian manifolds. Then there is a natural (pointwise) differentiable map $H^{1}(f): H^{1}(I, M) \longrightarrow H^{1}(I, N)$ where $H^{1}(f)(c)(t)=f(c(t)), t \in[0,1]$. Moreover $T H^{1}(I, M)=H^{1}(I, T M)$ that is $H^{1}$ commutes with the functor $T$ and for the smooth map $g: L \longrightarrow M, H^{1}(f \circ g)=H^{1}(f) \circ H^{1}(g)$ [9, 12].

For any (semi)spray $S$ on $M$ we claim that

$$
H^{1} S: T H^{1}(I, M) \longrightarrow T^{2} H^{1}(I, M)
$$

is a (semi)spray on $H^{1}(I, M)$ and $\left(H^{1} S\right)^{c}=H^{1}\left(S^{c}\right)$.

To this end, we first show that $H\left(\kappa_{i}\right)$ is the involution map of $T^{i} H^{1}(I, M)$, $i \in \mathbb{N}$. For the case $i=1$ the problem is trivial. Suppose that $i \geq 2$ and $\gamma:(-\epsilon, \epsilon)^{2} \longrightarrow T^{i-2} H^{1}(I, M)=H^{1}\left(I, T^{i-2} M\right)$ be a smooth curve and $k_{i}^{H^{1}}: T^{i} H^{1}(I, M) \longrightarrow T^{i} H^{1}(I, M)$ be the involution map that is

$$
k_{i}^{H^{1}} \partial_{s_{1}} \partial_{s_{2}} \gamma\left(s_{1}, s_{2}\right)=\partial_{s_{2}} \partial_{s_{1}} \gamma\left(s_{1}, s_{2}\right) \text {. }
$$

Then, for any $t \in[0,1]$ we have

$$
\begin{aligned}
\left(H^{1} \kappa_{i} \partial_{s_{1}} \partial_{s_{2}} \gamma\left(s_{1}, s_{2}\right)\right)(t) & =\kappa_{i} \partial_{s_{1}} \partial_{s_{2}} \gamma\left(s_{1}, s_{2}, t\right) \\
& =\partial_{s_{2}} \partial_{s_{1}} \gamma\left(s_{1}, s_{2}, t\right) \\
& =\partial_{s_{2}} \partial_{s_{1}} \gamma\left(s_{1}, s_{2}\right)(t)
\end{aligned}
$$


that is $H^{1} \kappa_{i}=\kappa_{i}^{H^{1}}$.

As a consequence $\kappa_{2}^{H^{1}} \circ H^{1} S=\left(H^{1} \kappa_{2}\right) \circ\left(H^{1} S\right)=H^{1}\left(\kappa_{2} \circ S\right)=H^{1} S$. Moreover,

$$
\begin{aligned}
\left(H^{1} S\right)^{c} & =D \kappa_{2}^{H^{1}} \circ \kappa_{3}^{H^{1}} \circ D H^{1} S \circ \kappa_{2}^{H^{1}} \\
& =D H^{1} \kappa_{2} \circ H^{1} \kappa_{3} \circ D H^{1} S \circ H^{1} \kappa_{2} \\
& =H^{1} D \kappa_{2} \circ H^{1} \kappa_{3} \circ H^{1} D S \circ H^{1} \kappa_{2} \\
& =H^{1}\left(D \kappa_{2} \circ \kappa_{3} \circ D S \circ \kappa_{2}\right) \\
& =H^{1}\left(S^{c}\right) .
\end{aligned}
$$

Consequently $\left(H^{1} S\right)^{c_{i}}=H^{1}\left(S^{c_{i}}\right)$ for any $i \in N$. On the other hand, $T^{i} H^{1}(I$, $M)=H^{1}\left(I, T^{i} M\right)$. These last two equalities motivate us to define

$$
T^{\infty} H^{1}(I, M)=\lim _{\longleftarrow} T^{i} H^{1}(I, M)
$$

and $H^{1} S^{c_{\infty}}:=\left(H^{1} S\right)^{c_{\infty}}$. However, we are not sure about the existence a reasonable manifold structure on $H^{1}\left(I, T^{\infty} M\right)$ since the manifold structure in the classical case (i.e. $H^{1}(I, N)$ ) deeply depends on the notion of the exponential mapping (and the Riemannian metric) on the target manifold which is not guaranteed in our case [8, 11]. In fact, on Fréchet modeled manifolds, an inverse function theorem is not always available and only under certain conditions (like tameness [11]) a version of the inverse function theorem (and consequently an exponential mapping) is available. Since for any $i \in \mathbb{N}, H^{1}\left(I, T^{i} M\right)=T^{i} H^{1}(I, T M)$ we can also define

$$
H^{1}\left(I, T^{\infty} M\right):=T^{\infty} H^{1}(I, T M) .
$$

Using the terminologies of Lang [13] or Klingenberg [12], one can show that if $S$ is a spray then $H^{1} S$ is also an spray. Now, proposition 3.13 implies that $H^{1} S^{c}=\left(H^{1} S\right)^{c}$ is a spray on $T H^{1}(I, M)=H^{1}(I, T M)$ and $H^{1} S^{c_{i}}=\left(H^{1} S\right)^{c_{i}}$, for any $i \in \mathbb{N}$.

Finally, using remark 3.1 and the techniques of proposition 4.1 of [19], enable us to lift a connection form the finite dimensional manifold $M$ to the manifold $T^{i} H^{1}(I, M), i \in \mathbb{N} \cup\{\infty\}$.

\section{REFERENCES}

[1] M.C. Abbati, A. Mania, On differential structure for projective limits of manifolds, J. Geom. Phys. Vol. 29, (1999) pp. 35-63

[2] R. Abraham, J.E. Marsden and T. Ratiu, Manifolds, Tensor Analysis, and Applications, Springer-Verlag, 2007.

[3] M. Aghasi and A. Suri, Splitting theorems for the double tangent bundles of Fréchet manifolds. Balkan journal of geometry and its applications, No. 15-2, 2010, 1-13.

[4] A. Ashtekar and J. Lewandowski, Differential geometry on the space of connections via graphs and projective limits, J. Geom. Phys. Vol. 17, No. 3 (1995) pp. 191-230.

[5] I. Bucataru, M.F. Dahl, A complete lift for semisprays, Int. J. Geom. Methods Mod. Phys. 7 (2), 2010, 267-287.

[6] I. Bucataru and M.F. Dahl, k-parameter geodesic variations, J. Geom. Phys. Vol. 62, 2012, 2121-2132.

[7] Del Riego, L. and Parker, P.E., Geometry of nonlinear connections, J. Nonlinear Analysis, 63, 2005, 501-510.

[8] H.I. Eliasson, Geometry of manifolds of maps, J. Differential Geom. 1, 1967, 169-194. 
[9] P. Flaschel and W. Klingenberg, Riemannsche Hilbertmannigfaltigkeiten. Periodische Geodätische, Lecture Notes in Mathematics 282, Berlin-Heidelberg-New York, Springer-Verlag, 1972.

[10] G.N. Galanis, Differential and Geometric Structure for the Tangent Bundle of a Projective Limit Manifold, Rendiconti del Seminario Matematico di Padova, Vol. 112, 2004.

[11] R.S. Hamilton, The inverse functions theorem of Nash and Moser, Bull. of Amer. Math. Soc., 7, 1982, 65-222.

[12] W. Klingenberg, Riemannian geometry, Walter de Gruyter, 1995.

[13] S. Lang, Fundamentals of differential geometry, Graduate Texts in Mathematics, vol. 191, Springer-Verlag, 1999.

[14] A. Kriegel, P.W. Michor, The convenient Setting of Global Analysis, AMS Mathematical Surveys and Monographs, 53, 1997.

[15] R. Miron, The geometry of higher order Lagrange spaces applications to Mechanics and Physics, Kluwer Academic publishers, 1997.

[16] A. Morimoto, Liftings of tensor fields and connections to tangent bundles of higher order, , Nagoya Math. J., Vol. 40, 1970, 99-120.

[17] O. Müller, A metric approach to Fréchet geometry, J. Geom. Phys., Vol. 58 (2008), pp. 1477-1500.

[18] H. H. Schaefer, Topological Vector Spaces, Springer-Verlag, 1971.

[19] A. Suri and M. Aghasi, Connections and Second order differential equations on infinite dimensional manifolds, Int. Electron. J. Geom., 6, (2), 2013, 45-56.

[20] A. Suri, Higher order tangent bundles, http://arxiv.org/abs/1403.3111v1, 2014.

[21] A. Suri, Isomorphism classes for higher order tangent bundles, http://arxiv.org/abs/1412.7321v1, 2014.

[22] F. Takens, A global version of the inverse problem of the calculus of variation, J. Diff. Geom., 14, 1979, 543-562.

[23] K. Yano and S. Ishihara, Tangent and Cotangent Bundles , Marcel Dekker, Inc., 1973.

[24] K. Yano and S. Kobayashi, Prolongations of tensor fields and connections to tangent bundles, I. General theory, J. Math. Soc. Japan, 18, 1966 194210.,

Department of Mathematics, Faculty of Sciences, Bu-Ali Sina University, HAMEDAN 65178, IRAN

E-mail address: a.suri@basu.ac.ir \& a.suri@math.iut.ac.ir \& ali.suri@gmail.com

Department of Mathematics, Faculty of Sciences, Bu-Ali Sina University, HAMEDAN 65178, IRAN

E-mail address: rastegarzade.math@yahoo.com 